\title{
Clinical Significance of Personalized Neoantigen Vaccine (G1-PES) for The Treatment of Various Types of Advanced Metastatic Cancer Patients in 2001 to 2014: Treatment Case Reports
}

John A Catanazaro ${ }^{1 *}$, Md Shamsuddin Sultan Khan ${ }^{1}$, Mohamed Khadeer Ahmded Basheer ${ }^{2}$, Anton Yuryev ${ }^{1}$, Andrew Dickens ${ }^{1}$

\begin{abstract}
Immunopeptide therapy has provided significant clinical improvements in the treatment of several malignancies. The generation 1 personalized edited sequence (G1-PES) vaccine administered to 43 severe metastatic cancer patients (terminal stage), safely and effectively in Dr. Catanzaro's clinic in 2001 to 2014. These all patients were considered for 3 to 4 months life support with no hope. Patients were on G1-PES an 18-month therapy program with the objective of achieving remission and cancer free survival within 18 months. Typically, patients received 4 cycles every 12 weeks. The safety and efficacy were assessed through adverse events, progression-free survival (PFS), overall survival (OS) and other parameters. Patients that received G1-PES were free from any serious adverse effects (SAE's), while receiving and after therapy. Typical reactions included slight fever, flu-like symptoms for 1-2 days and rash at route of administration site that lasted for 2-3 days. All of these minor reactions were self-limiting. Patients had significantly improved quality of life within 1-3 weeks of receiving therapy, with dimini-
\end{abstract}

Significance | Neoantigen cancer vaccine is highly effective to treat the various types of cancer which is conducted in 2001 to 2014 at Health and Wellness Institute Integrative Cancer Treatment under the direction of Dr. Catanzaro and his team of physicians.

${ }^{*}$ Correspondence: John catanzaro at Neo7logix, LLC, 539 W. Commerce St \#2886 Dallas, TX 75208. Email: john.catanzaro@neo7logix.com

Edited by Aman Shah Abdul Majid, Quest International University, Malaysia. And accepted by the Editorial Board August 06, 2020 (received for review July 05, 2020) -shing symptoms associated with their cancer and clinical evidence of cancer regression $(p<0.001)$. G1-PES vaccine was feasible and safe for patients with advanced metastatic cancer. G1-PES vaccine was designed based on $\mathrm{T}$ cell-mediated immune response targeting tumor neoantigens as antitumor efficacy.

Key words: Immunotherapy, Cancer, G1-PES (Generation-1 Personalized Edited Sequence), Neoantigen, personalized vaccine, peptide vaccine, advanced malignant tumor

\section{Introduction}

Malignant tumors are associated with high levels of morbidity and mortality worldwide with a reported 18.1 million new cases of cancer and 9.6 million cancer-related deaths in 2018 (Bray et al., 2018). The use of immunotherapeutic personalized vaccine is highly safe, effective and no-toxic for patients with many different types of cancers (Yoshida K. et al., 2011). There is no toxicities has been reported against the short peptide immunotherapy and many papers have been published for the potency of such peptides. In recent years, several clinical trials demonstrated that neo-antigens

\footnotetext{
Author Affiliation:

${ }^{1}$ Neo7logix USA LLC, 539 W. Commerce St \#2886 Dallas, TX 75208

${ }^{2}$ Neo7logix Australia Pty Ltd, 217 Flinders Street, Adelaide SA 5000
}

\section{Please cite this article:}

John Catanzaro, Md Shamsuddin Sultan Khan, Mohamed Khadeer Ahmded Basheer, Anton Yuryev, Andrew Dickens. (2020). Clinical Significance of Personalized Neoantigen Vaccine (G1-PES) for The Treatment of various Types of Advanced Metastatic Cancer Patients in 2001 to 2014: Treatment case reports. Journal of Precision Biosciences, 2(1), 106-115.

(C) 2019 BIOSCIENCES, a publication of Eman Research Ltd, Australia. This is an open access article under the CC BY-NC-ND license. (http://creativecommons.org/icenses/by-nc-nd/4.0/). (https://publishing.emanresearch.org) 
can facilitate response to checkpoint inhibition (Guo et al., 2018), thus dramatically altering our view on the clinical potential of neoantigens in cancer immunotherapy.

As such, neo-antigens are used to specifically direct the immune response towards cancer cells, thus minimizing the risk of developing autoimmune reaction against a patient's healthy tissue (Wang et al., 2015). On the other hand, self-antigens are used as adjuvants to boost the immune response and increase the duration of the immunity, thus minimizing the dose of antigen needed (Guo et al., 2018; Khong et al., 2016). The best self-antigens are the proteins whose expression is upregulated in tumor cells compared to normal cells (Yarchoan et al., 2017).

Studies have shown that the recognition of neo-antigens is an important driver of the clinical efficacy for standard-of-care cancer immunotherapies, whether they be via T-cell checkpoint blockade and/or adoptive T-cell therapy (CAR-T) (Yarchoan et al., 2017; Peng et al., 2019). In fact, the relevance of personalized neoantigens in tumor control and the biological properties of these antigens has been extensively studied (Yuryev and Catanzaro, 2019).

Crucially, recent technological advances can now be utilized to identify novel neo-antigens and to isolate $\mathrm{T}$-cells that recognize them in individual patients (Yuryev et al., 2019a, Peng et al., 2019). Analysis and Selection of the high confidence autologous gene products and associated proteins with up-regulation of cancer and progressive metastatic disease is crucial in identification of related matched peptides as therapeutic end products in the treatment of cancer. This personalized neo-antigen design has been employed in clinical interventions and we have treated about 500 patients with this kind of design in Health and Wellness Institute Integrative Cancer Treatment (HWIIC).

In principle, the G1-PES vaccine showed a broad anti-tumor efficacy as neoantigen vaccine could not be limited to certain tumor type as long as appropriate tumor neoantigens were identified. In this paper G1-PES (Generation-1 Personalized Edited Sequence) vaccine demonstrated the anti-tumor efficacy of neoantigen vaccine in various types of advanced metastatic cancer. The results indicated G1-PES vaccine could elicit specific $\mathrm{T}$ cell activation and induce broad spectrum of anti-tumor effects, without limitation to tumor type.

\section{Case Presentation}

This was an observation based clinical study at Health and Wellness Institute Integrative Cancer Treatment (HWIIC) in USA. In this study, we reported the post treatment effectiveness of the G1-PES vaccine. This study was a clinical treatment study inhouse. The treatment was provided by the clinical practitioner based upon the patient diagnosis, approved by Internal Review Committee and Independent Ethics Committee and conducted in accordance with Declaration of Helsinki and the International Conference on Harmonization Guidelines for Good Clinical Practice. All patients had signed informed consent forms before treatment. The primary endpoints of the treatment were safety and feasibility. And the secondary endpoints were efficacy based on PFS, OS and neoantigen-specific immune responses. The safety of the study was assessed on the basis of occurrence of adverse events (AEs). The feasibility of this observation study was assessed by whether neoantigen could be identified and the vaccines could be synthesized for clinical use.

The patients were (age 40 to 70 years old) terminal stage with extensive metastatic cancer who has been treated between 2001 to 2014 in HWIIC. These patients were found to have Breast Cancer, Colon Cancer, Prostate Cancer, Malignant Melanoma, Multiple Myeloma, Leiomyosarcoma, Non Small Cell Lung Carcinoma, Squamous Cell Carcinoma Neck, Lymphoma, Brain Cancer, Bladder Cancer, Astrocytoma, Ovarian Cancer, Schwanomma, Leukemia, Angiosarcoma, Esophageal Cancer and Liposarcoma (Table 1). A positron emission tomography (PET) scan in February 2014 did not show any evidence of malignancy.

The patients were received daily $1 \mathrm{mg}$ of G1-PES for 21 days in each cycle in total of 4 cycles over an 18 month period. We have reported for 43 patients who showed significant improvement in disease states. G1-PES comprised of 16 20 short length amino acid peptides. The peptides were pooled as single pool (based on HLA typing, affinity and allele frequency) and then injected subcutaneously (s.c.) at a quantity of $1 \mathrm{mg}$ per peptide in upper arms and para-umbilical area respectively. Patients were scheduled to receive G1-PES with Poly ICLC, or MF59 (46 $\mu \mathrm{g})$ as adjuvant for 21 days (i.e. 1st cycle), in total of four cycles over an 18 months period. PBIMA-G1-PES was developed by Dr. Catanzaro and his team through Harvard / Dana Farber Proteomics Bioinformatics Laboratories and NeoBioLabs in Boston. The design for patients is true personalization based upon the patient's cancer diagnosis in 2001 to 2014.

Clinical assessment, monitoring and follow-up during the treatment were conducted, including physical examination, ECOG performance, vital sign, blood test, urinalysis to assure the safety of each immunization; imaging examination at baseline and approximately every 4 months post-vaccination to assess clinical efficacy; and serum electrophoresis (antibodies), inflammation markers, cancer biomarkers (tumor antigens), flow cytometry ( $\mathrm{T}$ 
cell subsets and cytokines) conducted pre-treatment and every 8 12 weeks after treatment for the detection of specific immune response. The related adverse events (AEs) were recorded and graded for safety evaluation according to the National Cancer Institute Common Terminology Criteria for Adverse Events (version 4.0) throughout whole treatment period.

\section{Design of G1-PES personalized neoantigen vaccines}

To identify mutations derived neoantigens, some tumor tissues and blood samples were obtained from cancer patients by surgery, biopsy or intravenous blood sampling with proteomics, bioinformatics sand HLA compatibility. In case of unavailability of tumor fresh sampling, formalin-fixed paraffin-embedded (FFPE) samples were used instead.

The bioinformatics analysis, which consists of proteomics sequencing of normal tissue/cells and tumor cells and urine for neoantigen's precision mapping, HLA affinity binding, prediction, ranking and selection, was performed by PBIMA/G1-PES formula. The personalized neoantigen peptides were manufactured in the cGMP facility standard (bacteria-free, TFA free, $>95.0 \%$ purity and quantities of bacterial endotoxin less than $1 \mathrm{EU} / \mathrm{mg}$ ) using chemical synthesis. The solubility of peptides was determined during synthesis to select the best diluent for vaccine pool.

\section{Results}

The results of G1-PES is shown below after receiving treatment. The treated patients showed average, $58.37 \pm 32.25 \%$ tumor regression after treatment (Figure 1) without any adverse effect (Table 2). The average extended survival was recorded $5.78 \pm 2.7$ years in overall cancer patients (Figure 2). The result showed average, $7.3 \pm 2.5$ score for quality of life in overall cancer patients on the 10-points scoring scale (Figure 3). Among 43 patients, 14 patients showed $100 \%$ tumor regression. While majority of the other patients displayed tumor regression in a range between 40 to $80 \%$. Among all the types of cancers treated, lymphoma patients showed significantly $(\mathrm{p}<0.001)$ highest response with $90.0 \pm 14.1 \%$ tumor regression followed by the prostate cancer patients $(\mathrm{p}<0.01)$ with $80.0 \pm 28.3 \%$ tumor regression. The patients with squamous Cell Carcinoma of Neck ( $65.0 \pm 49.5 \%)$, multiple myeloma (60.7 \pm $31.7 \%)$, malignant melanoma $(68.8 \pm 37.5 \%)$ and breast cancer $(50$ $\pm 24.5 \%$ ) showed moderate tumor regression response (Figure 4). The patients with non-small cell lung carcinoma $(40.0 \pm 0.0 \%)$ and colon cancer $(42.0 \pm 36.3 \%)$ showed statistically significant $(\mathrm{p}<0.1)$ tumor regression effects (Figure 5). Whereas, the patients with leiomyosarcoma $(26.7 \pm 11.5 \%)$ and brain cancer $(25.0 \pm 0.0 \%)$ showed mild turmor regression effects. Among all the cancer types treated, patients with lymphoma demonstrated significantly $(p<0.005)$ highest extended survival rate of $6.0 \pm 5.7$ years (Figure 6). Whereas, the other types of cancer showed a range between 1.5 to 3.9 years of extended survival. Among all the different types of cancer patients treated, the patients with lymphoma demonstrated significantly $(\mathrm{p}<0.001)$ improved quality of life with $10.0 \pm 0.0$ scores (Figure 7). Similarly, the patients with squamous cell carcinoma of neck showed $9.0 \pm 0.0$ score and the breast cancer patients showed $8.6 \pm 0.5$ scores for quality of life. Patients with the other cancer types also demonstrated statistically significant improvement in quality of life with the score range between 6 to 7.5. However, the patients with brain cancer showed mild improvement in quality of life with a score of $4.5 \pm 0.7$. All the patients with lymphoma and squamous cell carcinoma of neck demonstrated complete regression of tumor without recurrence. Whereas, 20 to $50 \%$ of patients with other cancer type showed recurrence of tumors after stopping the therapy (Table 2, Figure 8).

\section{Discussion}

We have directly shown the ability of G1-PES vaccine, precision personalized immunotherapy to target different types of cancer patients. We have the list of over 500 patients who received this personalized therapy under their consent from 2001 to 2014 at the Dr. Catanzaro's clinic HWIIC and his treatment protocol. There were no serious adverse events experienced by any of the patients in these studies which have demonstrated the safety and efficacy of this personalized vaccine.

The Health and Wellness Institute Integrative Cancer Treatment (HWIIC) and Cancer Research Group in Seattle administered individualized isolated immunopeptides to more than 500 cancer patients, safely and effectively, as a form of adjunctive treatment in cooperation with other conventional treatments required by the patient's oncologist.

Patients in HWIIC were on an 18-month therapy program with the objective of achieving remission and cancer free survival within 18 months. Typically, patients received 4 cycles of their own isolated immunopeptides every 12 weeks. These peptides can be administered orally, intranasally and sublingually; routes of administration are determined by the scope of practice criteria.

Patients that received their own immunopeptides were free from any serious adverse effects (SAE's), while receiving and after therapy. Typical reactions included slight fever, flu-like symptoms for 1-2 days and rash at route of administration site that lasted for 2-3 days. All of these minor reactions were self-limiting. Patients had significantly improved quality of life within 1-3 weeks of receiving therapy, with diminishing symptoms associated with their cancer and clinical evidence of cancer regression. In accordance with our findings, recent clinical studies on neoantigens personalized vaccine demonstrate that it is an effective anti-cancer treatment that helps to improve vitality and quality of life of cancer patients who are failed on conventional therapy, chemotherapy, surgery and radiotherapy. The data shows 
Table 1 | Demographic and disease characteristics at baseline

\begin{tabular}{|c|c|}
\hline Characteristics & Patients $(\mathrm{N}=43)$ \\
\hline \multicolumn{2}{|l|}{ Age (yr) } \\
\hline Mean & $59 \pm 10$ \\
\hline Range & $40-70$ \\
\hline \multicolumn{2}{|l|}{ Age category-no. (\%) } \\
\hline$\geq 50$ yrs. & 16 \\
\hline$\leq 50$ yrs & 6 \\
\hline \multicolumn{2}{|l|}{ Sex-no. (\%) } \\
\hline Male & $13(30 \%)$ \\
\hline Female & $30(70 \%)$ \\
\hline \multicolumn{2}{|l|}{ Metastatic sites-no. (\%) } \\
\hline Bone & $80 \%$ \\
\hline Blood & $90 \%$ \\
\hline Brain & $55 \%$ \\
\hline Liver & $75 \%$ \\
\hline Lung & $75 \%$ \\
\hline Mediastinum & $75 \%$ \\
\hline \multicolumn{2}{|l|}{ ECOG performance-status score-no. $(\%)^{\star}$} \\
\hline 0 & 0 \\
\hline 1 & 43 \\
\hline \multicolumn{2}{|l|}{ Radiotherapy-no. (\%) } \\
\hline Yes & $70 \%$ \\
\hline No & $30 \%$ \\
\hline \multicolumn{2}{|l|}{ Lines of prior systematic therapy-no. (\%) } \\
\hline 30 & $75 \%$ \\
\hline 13 & $25 \%$ \\
\hline \multicolumn{2}{|l|}{ Tumor type-no. (\%) } \\
\hline Breast Cancer (Female) & 7 \\
\hline Colon Cancer (three Female, two Male) & 5 \\
\hline Prostate Cancer (Male) & 5 \\
\hline Malignant Melanoma (two Male, two Female) & 4 \\
\hline Multiple Myeloma (one Female, two Male) & 3 \\
\hline Leiomyosarcoma (one Male, two Female) & 3 \\
\hline Non Small Cell Lung Carcinoma (Female) & 2 \\
\hline Squamous Cell Carcinoma Neck (Male) & 2 \\
\hline Lymphoma (Male) & 2 \\
\hline Brain tumor (Female) & 2 \\
\hline Bladder Cancer (Male) & 1 \\
\hline Astrocytoma (Female) & 1 \\
\hline Ovarian Cancer (Female) & 1 \\
\hline Schwanomma (Female) & 1 \\
\hline Leukemia (Female) & 1 \\
\hline Angiosarcoma (Female) & 1 \\
\hline Esophageal Cancer (Male) & 1 \\
\hline Liposarcoma (Female) & 1 \\
\hline
\end{tabular}

${ }^{\star}$ Eastern Cooperative Oncology Group (ECOG) performance-status scores range from 0 to 5, with 0 indicating no symptoms and higher scores indicating increasing disability. 
Table 2 | Treatment related AEs in all treated patients

\begin{tabular}{|l|l|l|l|l|}
\hline \multirow{2}{*}{} & \multicolumn{4}{|c}{ Patients } \\
\cline { 2 - 5 } & Any Grade & $\%$ & No. & $\%$ \\
\cline { 2 - 5 } & No. & 0 & 0 & 0 \\
\hline Any AE & 0 & 0 & 0 & 0 \\
\hline Fatigue & 2 & 0 & 0 & 0 \\
\hline Chill & 2 & 0 & 0 & 0 \\
\hline Slight-Fever & 2 & 0 & 0 & 0 \\
\hline Emesis & 0 & 0 & 0 & 0 \\
\hline Muscle soreness & 3 & 0 & 0 & 0 \\
\hline Injection site reaction & 3 & 0 & 0 & 0 \\
\hline Dizzy & 0 & 0 & 0 & 0 \\
\hline Nausea & 0 & 0 & 0 & 0 \\
\hline Upper gastrointestinal & 0 & 0 & 0 \\
\hline heamorrhage & 0 & 0 & 0 & 0 \\
\hline Lose weight & & 0 & 0 & 0 \\
\hline Acute allergic reaction & 0 & 0 & 0 \\
\hline
\end{tabular}

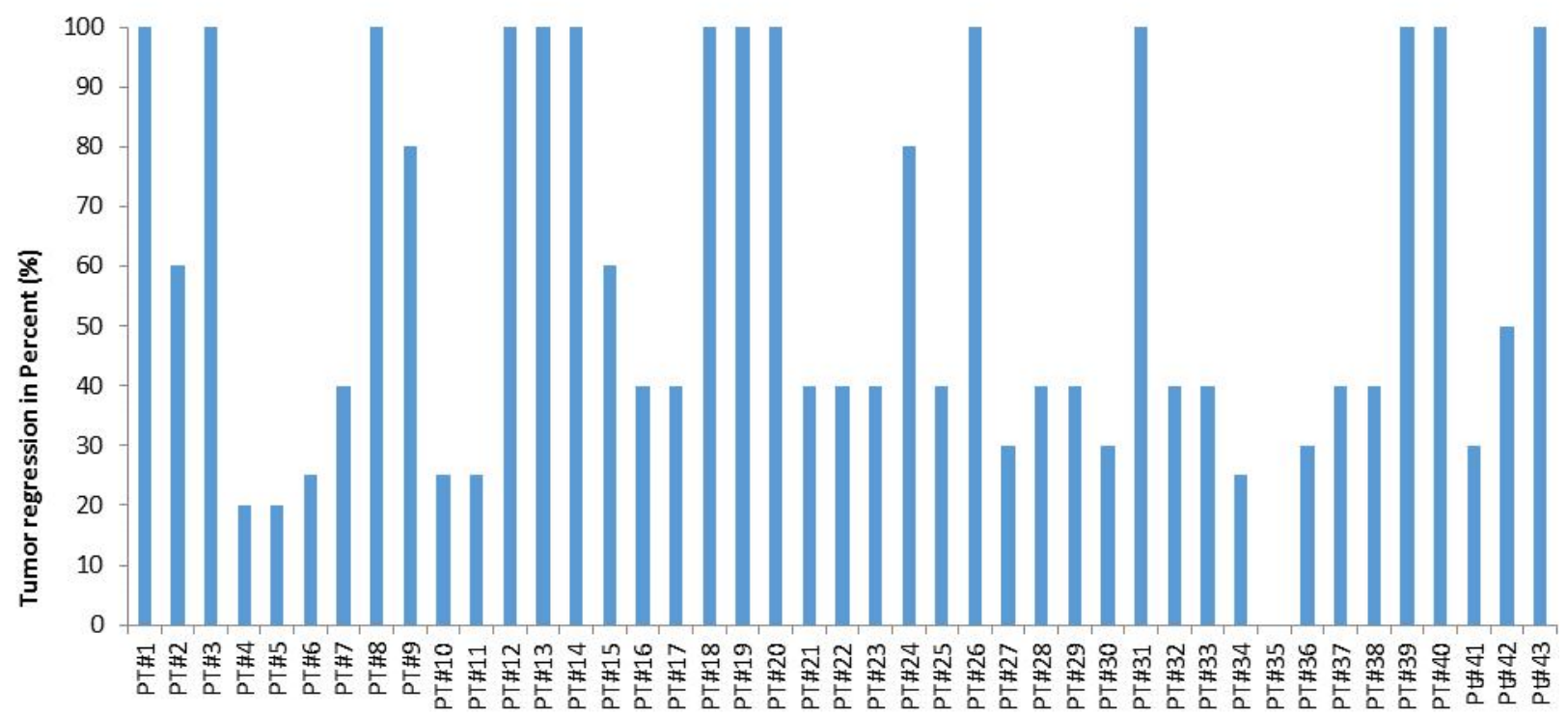

Patients

Figure 1 | Summary of treatment outcome from personalized treatment with G1-PES 
Table 3 | Percentage of tumor regression in patients.

\begin{tabular}{|l|l|}
\hline No. of Patients & Tumor Regression \\
\hline 14 & $100 \%$ \\
\hline 2 & $80 \%$ \\
\hline 2 & $60 \%$ \\
\hline 1 & $50 \%$ \\
\hline 14 & $40 \%$ \\
\hline 4 & $30 \%$ \\
\hline 4 & $25 \%$ \\
\hline 2 & $20 \%$ \\
\hline
\end{tabular}

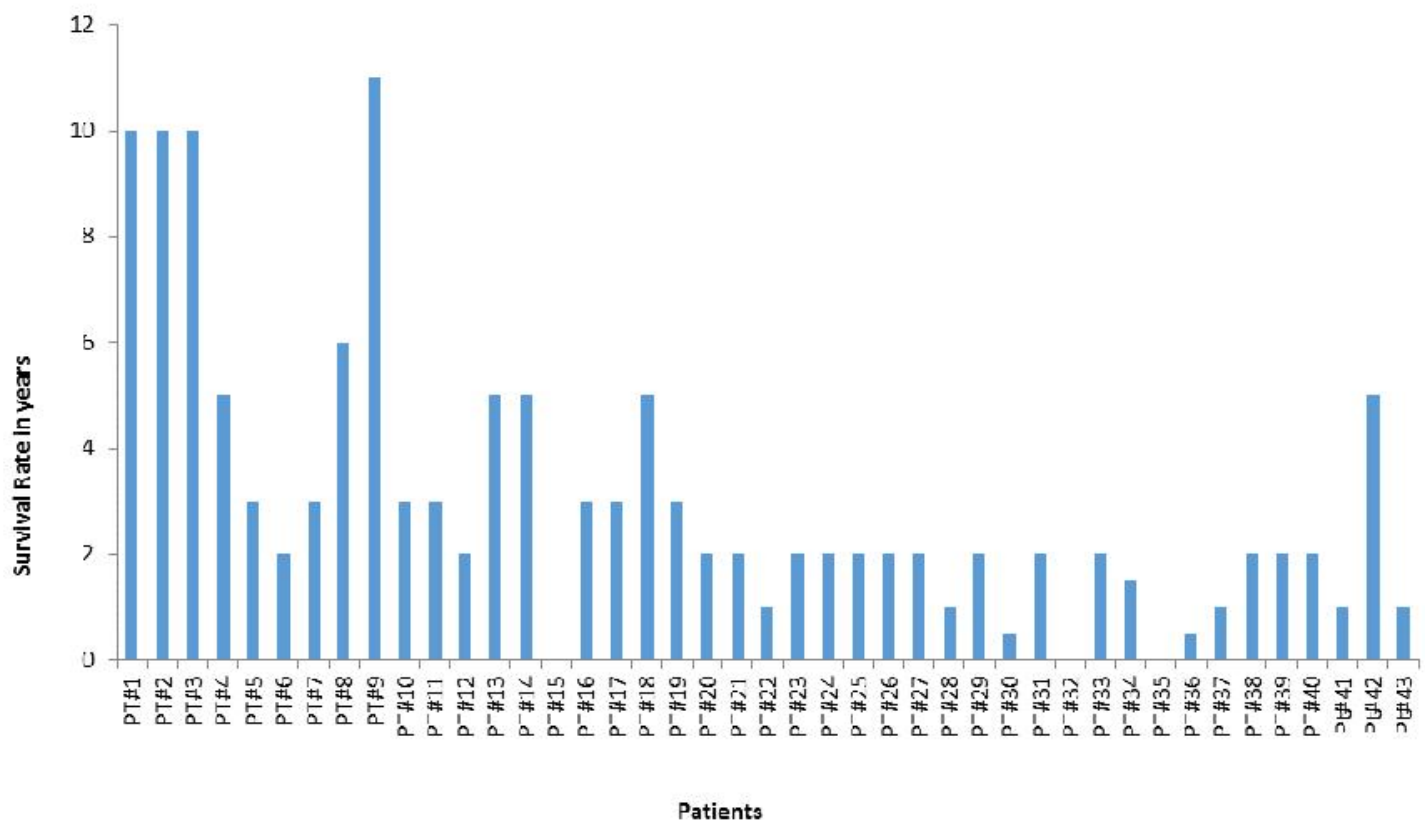

Figure 2 | Comparing the extended survival in patients

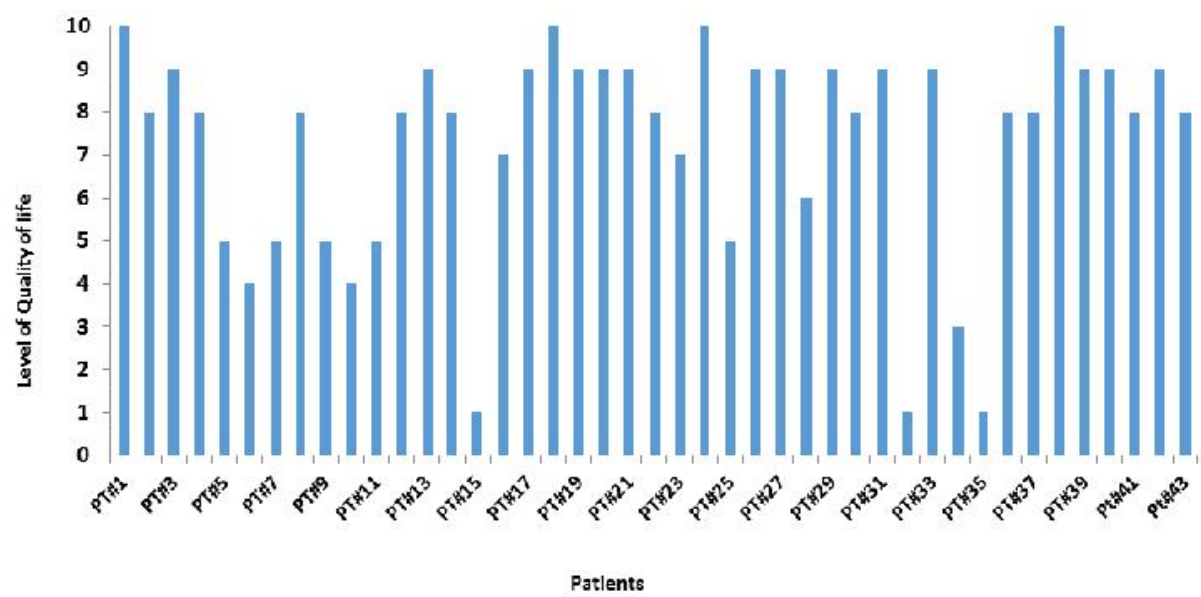

Figure 3 | Comparing Quality of Life in Patients (10 point scale) 


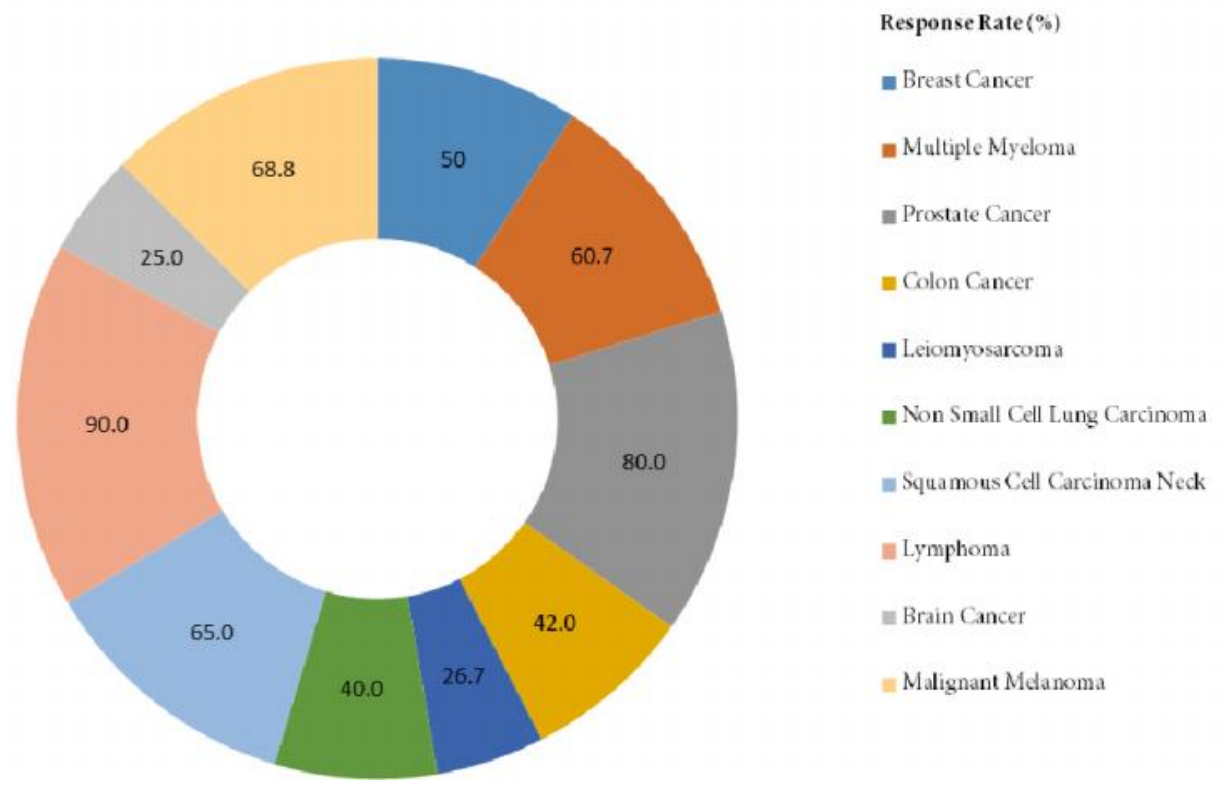

Figure 4 | Differential Response of Different Cancer Types to G1-PES Therapy

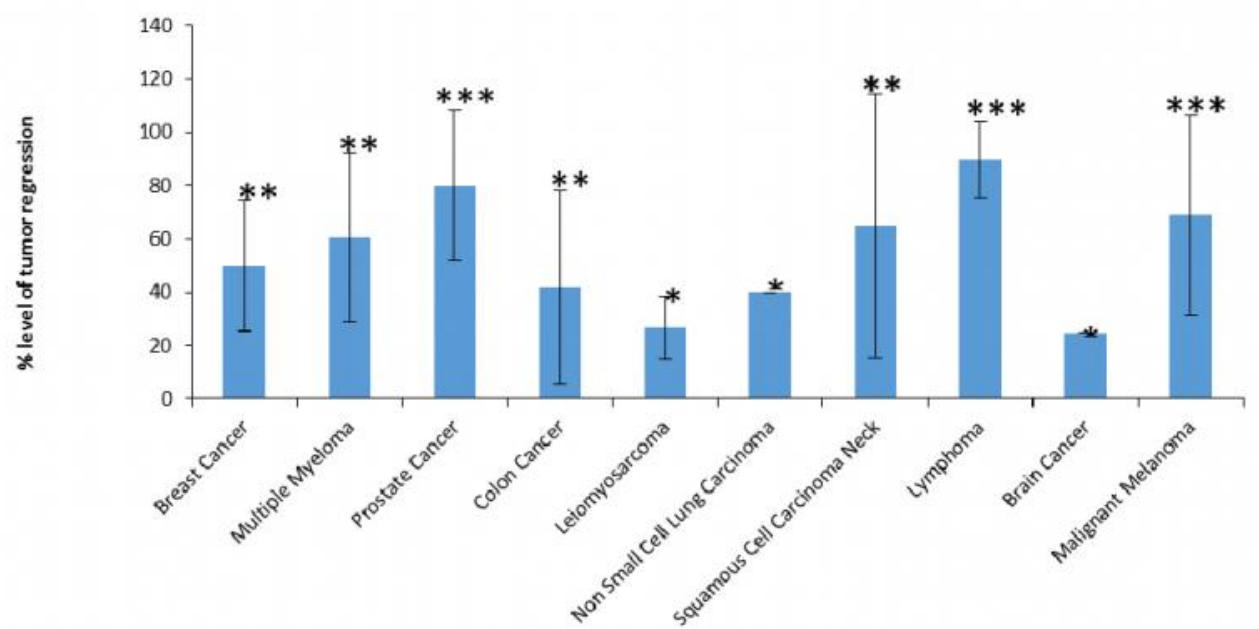

Figure 5 | Tumor Regression Effect of G1-PES on Different Tumor Types. G1-PES demonstrated $58.37 \pm 32.25 \%$ tumor regression in overall cancer patients.

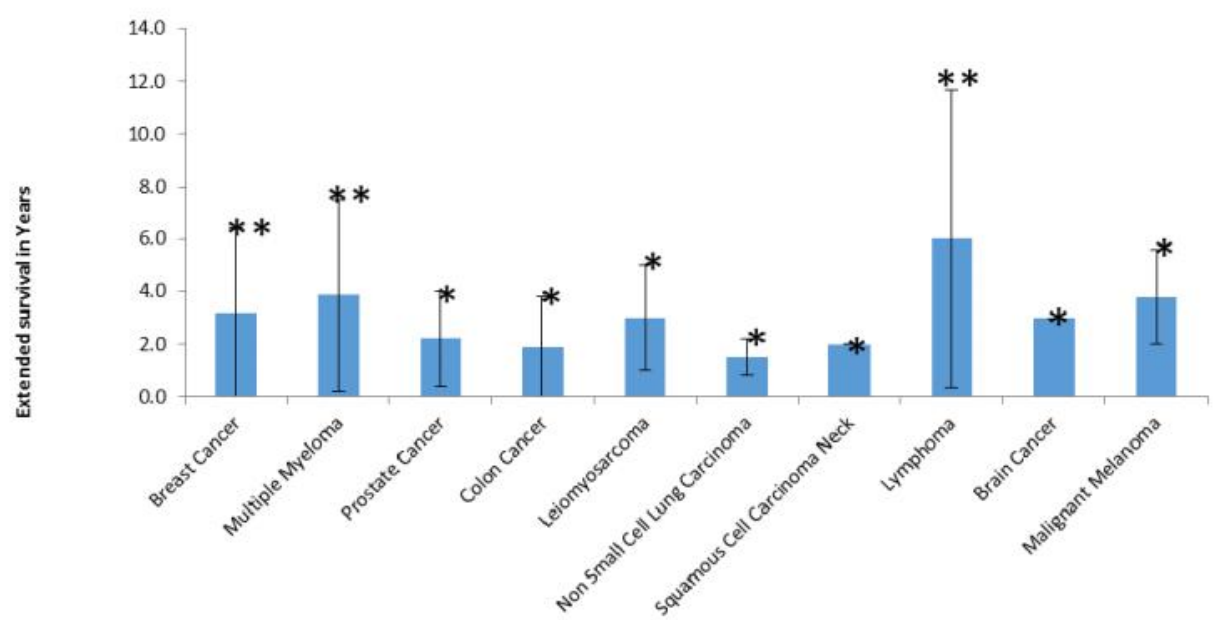

Figure 6 | Extended Survival of patients in years. G1-PES showed $5.78 \pm 2.7$ years of extended survival in overall cancer patients. 


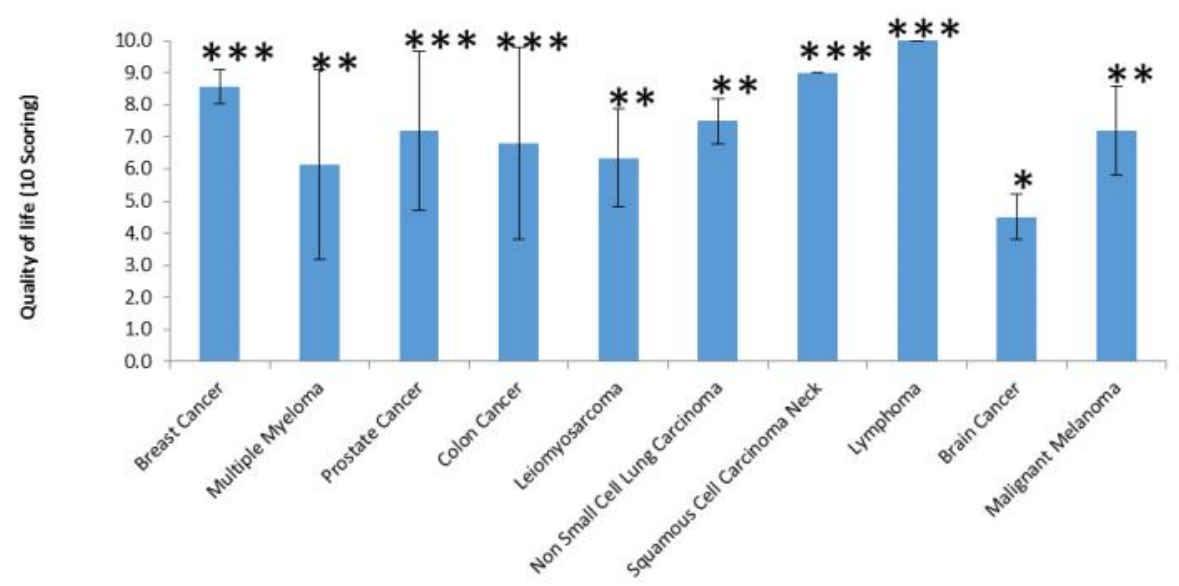

Figure 7 | Quality of life of patients based on extended survival. G1-PES treated patients scored $7.3 \pm 2.5$ for quality of life on the 10-points scoring scale.

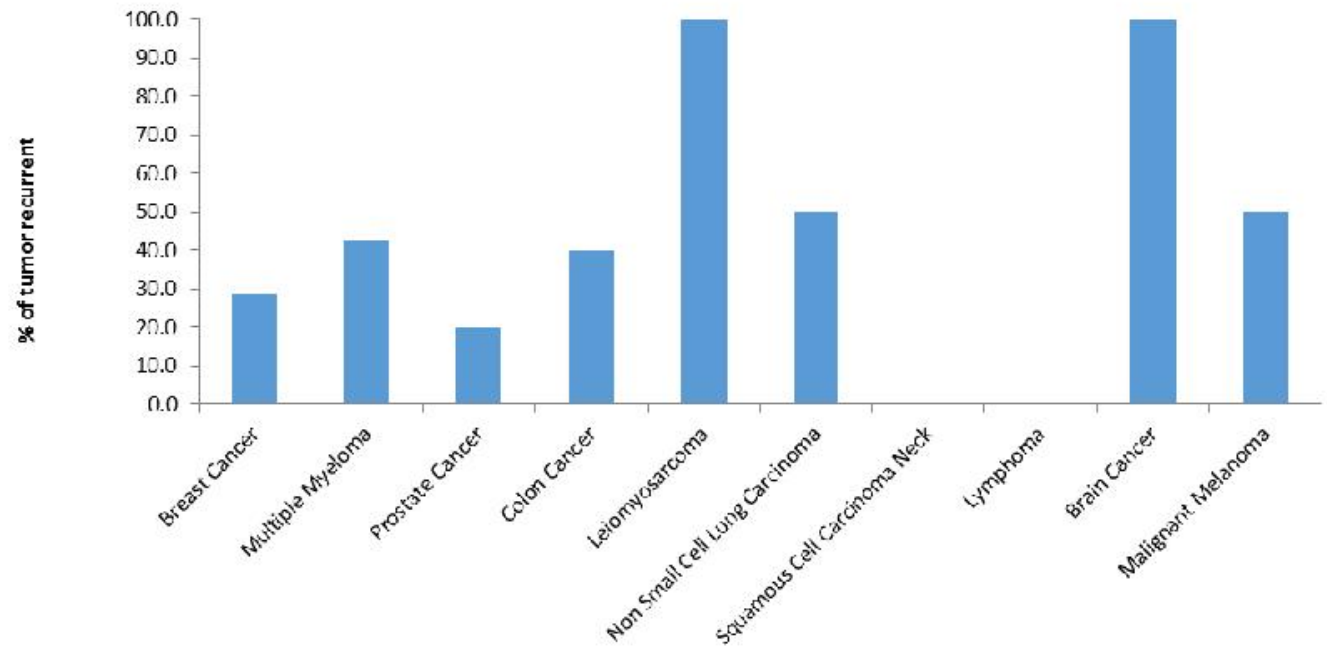

Figure 8 | Tumor Recurrence after G1-PES Therapy

complete tumor regression, with progression free survival of patient (Ott et al., 2017; Chen et al., 2019). The neoantigen personalized vaccination showed a low risk and incidence of SAEs in other study with 500 advanced cancer patients, including 174 prostate, 74 colon, 51 pancreatic and 43 gastric cancer patients (Yoshida K. et al., 2011). Moreover, favorable immune effects were observed with administration of personalized cancer peptide vaccines which included disease stability.

Patients had follow-up evaluations within 12 weeks on clinical progress through serology, specialized imaging (PET/MRI). Within 12 weeks of initiating treatment, patients typically were 65\% improved overall. Additional modalities were administered including and not limited to mind body energy, dietary, nutraceutical/bioceutical supplementation, physical and manual modalities, intravenous modalities including sodium bicarbonate (http://cancerres.aacrjournals.org/content/69/6/2260), DMSO, vitamin $\mathrm{C}$ and UV treatment in the therapy design along with the immunopeptides and are not contraindicated. Therapeutic modalities are defined and determined by the scope of practice criteria.

Patients receiving chemotherapy did not receive immunopeptides on days that they were receiving chemotherapy. Peptides were administered 2 days prior to chemotherapy administration and when patients were on aggressive daily chemotherapy cycles, immunopeptides were administered during a break in the chemotherapy treatment cycle.

Pharmacokinetic actions of personalized peptides are best seen by direct injection subcutaneously, intranodal, intravenously, intratumoral and intranasal for direct absorption and metabolism. Oral administration is not an optimal delivery due to alteration of 
peptide integrity by gastric and digestive activity of the gastrointestinal tract.

This case report has demonstrated the feasibility, safety and efficacy of G1-PES on patients with various types of advanced solid tumors. G1-PES monotherapy might show its efficacy through neoantigen-specific $\mathrm{T}$ cell response, high $\mathrm{T}$ cell infiltration into tumors and remodel tumor-immune microenvironment alone, suggesting its great potential as cancer immunotherapy. It is probably due to the fact that, G1-PES treatment can result in tumor necrosis, which will become an immunogenic source, providing pro-inflammatory signals (McGahan et al., 1992; Widenmeyer et al., 2011). After RFA, it may activate and/or generate a large amount of IFN- $\gamma$ and/or neoantigen, while improving the expression of other costimulating factors and the presenting of tumor antigen to $\mathrm{T}$ cells. This report provides proof-of-principle that a G1-PES vaccine can be produced and administered to a patient to generate highly specific immune responses against that individual's tumour. Additionally, the efficacy of G1-PES might be CD8+ and CD4+ T cells mediated tumour cell apoptosis (Tran, E. et al. 2014); Schumacher, T. et al. 2014) using the short peptides in the vaccine, leading to activation of both CD8+ and CD4+ $\mathrm{T}$ cells against tumor cells. Importantly, the proportion of tumour neoantigens inducing a T-cell response after vaccination might be due to nonantigen-directed immunotherapy, such as checkpoint blockade or tumour-infiltrating lymphocyte Therapy (Van Rooij et al. 2013; Rizvi et al. 2015; Linnemann et al. 2015; Prickett et al. 2016). Thus, in agreement with a reported melanoma study showing T-cell responses to a dendritic-cell-based neoantigen vaccine (Carreno et al. 2015), this case report demonstrates that a personal neoantigen vaccine broadens the repertoire of neoantigen-specific $\mathrm{T}$ cells substantially beyond what is induced by existing immunotherapeutics.

\section{Conclusion}

The treatment significance of G1-PES in terms of overall survival and progression-free survival has been reported. This is the first case report of multiple cancer patient in which G1-PES immunotherapy was evaluated with favorable antitumor response. In Conclusion, A summary of a random group of the patients treated shown significant efficacy for G1-PES. Among 43 patients, 14 patients showed $100 \%$ tumor regression. Majority of the other patients displayed tumor regression in a range between 40 to $80 \%$. Patients with lymphoma, prostate cancer, malignant melanoma, squamous cell carcinoma of neck and multiple myeloma demonstrated more pronounced response rate, tumor regression, extended survival life and improved quality of life. All the patients with lymphoma and squamous cell carcinoma of neck demonstrated complete regression of tumor without recurrence.
However, 20 to $50 \%$ of patients with other cancer type showed recurrence of tumors after stopping the therapy.

\section{Author Contribution}

M.S.S.K. wrote and revised the manuscript, A.Y., J.C. has reviewed the manuscript and M.K.A.B has analysed the clinical data and statistics.

\section{Acknowledgment}

We are thankful to all doctors and nurses who support during treatment formulation preparations and managing the patients at HWIIC.

\section{Competing financial interests}

The author(s) declare no competing financial interests.

\section{References}

Bray F., Ferlay J., Soerjomataram I., Siegel R.L., Torre L.A. and Jemal A. (2018), Global cancer statistics 2018: GLOBOCAN estimates of incidence and mortality worldwide for 36 cancers in 185 countries. CA Cancer J Clin, 68, 394-424. https://doi.org/10.3322/caac.21492 PMid:30207593

Carreno, B. M. et al. (2015). A dendritic cell vaccine increases the breadth and diversity of melanoma neoantigen-specific T cells. Science 348, 803-808.

https://doi.org/10.1126/science.aaa3828

PMid:25837513 PMCid:PMC4549796

Chen F., Wei J. and Liu B. (2019). Neoantigen identification strategies enable personalized immunotherapy in refractory solid tumors. J Clin Invest, 129(5):20562070.

https://doi.org/10.1172/JCI99538

PMid:30835255 PMCid:PMC6486339

Guo Y., Lei K., and Tang L. (2018). Neoantigen Vaccine Delivery for Personalized $\begin{array}{lllll}\text { Anticancer Immunotherapy. Front Immunol, } & 9, & 1499 .\end{array}$ doi:10.3389/fimmu.2018.01499.

https://doi.org/10.3389/fimmu.2018.01499

PMid:30013560 PMCid:PMC6036114

Khong, H. and Overwijk, W.W. (2016). Adjuvants for peptide-based cancer vaccines. Journal for Immunotherapy of Cancer, 4(56). doi:10.1186/s40425-016-0160-y. https://doi.org/10.1186/s40425-016-0160-y

PMid:27660710 PMCid:PMC5028954

Linnemann, C. et al. (2015). High-throughput epitope discovery reveals frequent recognition of neo-antigens by CD4+ T cells in human melanoma. Nat. Med. 21, 8185

https://doi.org/10.1038/nm.3773

PMid:25531942 
McGahan JP, Brock JM, Tesluk H, Gu WZ, Schneider P, Browning PD. (1992). Hepatic ablation with use of radio-frequency electrocautery in the animal model. $J$ Vasc Interv Radiol, 3(2), 291-7. doi 10.1016/s1051-0443(92)72028-4.

https://doi.org/10.1016/S1051-0443(92)72028-4

Ott P. A., Hu Z., Keskin D. B., Shukla S. A., Sun J., Bozym D. J., Zhang W., Luoma A., Giobbie-Hurder A., Peter L., Chen C., Olive O., Carter T. A., Li S., Lieb D. J., Eisenhaure T., Gjini E., Stevens J., Lane W. J., Javeri, I. and Wu, C. J. (2017). An immunogenic personal neoantigen vaccine for patients with melanoma. Nature, 547(7662), 217-221

https://doi.org/10.1038/nature22991

PMid:28678778 PMCid:PMC5577644

Peng M., Mo Y., Wang Y., Wu P., Zhang Y., Xiong F., Guo C., Wu,X., Li Y., Li X., Li G., Xiong W. and Zeng Z. (2019). Neoantigen vaccine: an emerging tumor immunotherapy. Mol Cancer, 18, 128

https://doi.org/10.1186/s12943-019-1055-6

PMid:31443694 PMCid:PMC6708248

Prickett, T. D. et al. (2016). Durable complete response from metastatic melanoma after transfer of autologous T cells recognizing 10 mutated tumor antigens. Cancer Immunol. Res. 4, 669-678.

https://doi.org/10.1158/2326-6066.CIR-15-0215

PMid:27312342 PMCid:PMC4970903

Rizvi, N. A. et al. (2015). Mutational landscape determines sensitivity to PD-1 blockade in non-small cell lung cancer. Science 348, 124-128.

https://doi.org/10.1126/science.aaa1348

PMid:25765070 PMCid:PMC4993154

Schumacher, T. et al. (2014). A vaccine targeting mutant IDH1 induces antitumour immunity. Nature 512, 324-327.

https://doi.org/10.1038/nature13387

PMid:25043048

Tran, E. et al. (2014). Cancer immunotherapy based on mutation-specific CD4+ T cells in a patient with epithelial cancer. Science 344, 641-645 (2014)

https://doi.org/10.1126/science.1251102

PMid:24812403 PMCid:PMC6686185

Van Rooij, N. et al. (2013). Tumor exome analysis reveals neoantigen-specific T-cel reactivity in an ipilimumab-responsive melanoma. J. Clin. Oncol. 31, e439-e442. https://doi.org/10.1200/JC0.2012.47.7521

PMid:24043743 PMCid:PMC3836220

Wang S., Liu H., Zhang X. and Qian F. (2015). Intranasal and oral vaccination with protein-based antigens: advantages, challenges and formulation strategies. Protein Cell, 6(7), 480-503. https://doi.org/10.1007/s13238-015-0164-2

PMid:25944045 PMCid:PMC4491048

Widenmeyer M, Shebzukhov Y, Haen SP, Schmidt D, Clasen S, Boss A, et al. (2011) Analysis of tumor antigen-specific T cells and antibodies in cancer patients treated with radiofrequency ablation. Int J Cancer, 128(11), 2653-62 doi 10.1002/ijc.25601. https://doi.org/10.1002/ijc.25601

PMid:20715115

Yarchoan M., Johnson B.A., Lutz E.R., Laheru D.A. and Jaffee E.M. (2017). Targeting neoantigens to augment antitumour immunity. Nat Rev Cancer, 17, 209-22. 10.1038/nrc.2016.154

https://doi.org/10.1038/nrc.2017.74

https://doi.org/10.1038/nrc.2016.154

PMid:28233802 PMCid:PMC5575801

Yoshida K, Noguchi M, Mine T, Komatsu N., Yutani S., Ueno T., Yanagimoto H., Kawano K., Itoh K., and Yamada A. (2011). Characteristics of severe adverse events after peptide vaccination for advanced cancer patients: Analysis of 500 cases. Oncol Rep, 25(1):57-62.

https://doi.org/10.3892/or_00001041

PMid:21152877

Yuryev A. and Catanzaro J. (2019). Development of Personalized Therapeutics Using Neo7logix ${ }^{\circledR}$ Precision Profiling for Progressive CNS Inflammation and Autoimmune Disease. Biosciences, 1(1), 001-006.

https://doi.org/10.25163/biosciences.112087DB112921119

https://doi.org/10.25163/biosciences.112090DB112921119

Yuryev A., Catanzaro J., Khan, M. S. S. (2019a). Development of Personalized Therapeutics Using Neo7logix Precision Profiling in Lung Cancer. Biosciences, 1(1), 016-025.

https://doi.org/10.25163/biosciences.112090DB112921119

Submit your next manuscript to Journal of Precision Biosciences published by Eman Research Ltd

- Convenient online submission

- Thorough peer review

- No space constraints or color figure charges

- Immediate publication on acceptance

- Inclusion in Australian National Libraray and Google Schola

- Both Open (80-100\% subsidized APC by ER) \& non-open access option

Submit your manuscript at https://publishing.emanresearch.org 